\title{
Baseline Renal Function Predicts Hyponatremia in Liver Cirrhosis Patients Treated with Terlipressin for Variceal Bleeding
}

\author{
Sung Eun Kim, ${ }^{1}$ Dong Min Jung, ${ }^{1}$ Ji Won Park, ${ }^{1}$ Yeonmi Ju, ${ }^{1}$ Bohyun Lee, ${ }^{1}$ Hyoung Su Kim, \\ Ki Tae Suk, ${ }^{3}$ Myoung Kuk Jang, ${ }^{2}$ Sang Hoon Park, ${ }^{4}$ Jun Goo Kang, ${ }^{1}$ Jae Seung Soh, \\ Hyun Lim, ${ }^{1}$ Ho Suk Kang, ${ }^{1}$ Sung Hoon Moon, ${ }^{1}$ ChulSik Kim, ${ }^{1}$ SeongJin Lee, ${ }^{1}$ \\ Jong Hyeok Kim, ${ }^{1}$ Myung Seok Lee, ${ }^{4}$ Dong Joon Kim, ${ }^{3}$ Sung-Hee $\mathrm{Ihm}^{1}{ }^{1}$ and \\ ChoongKee Park ${ }^{1}$ \\ ${ }^{1}$ Department of Internal Medicine, Hallym University Sacred Heart Hospital, Hallym University College of Medicine, Anyang, \\ Republic of Korea \\ ${ }^{2}$ Department of Internal Medicine, Hallym University Gangdong Sacred Heart Hospital, Gangdong, Republic of Korea \\ ${ }^{3}$ Department of Internal Medicine, Hallym University Chuncheon Sacred Heart Hospital, Chuncheon, Republic of Korea \\ ${ }^{4}$ Department of Internal Medicine, Hallym University Gangnam Sacred Heart Hospital, Seoul, Republic of Korea \\ Correspondence should be addressed to Jun Goo Kang; kjg0804@empas.com
}

Received 18 January 2017; Revised 5 July 2017; Accepted 30 July 2017; Published 17 September 2017

Academic Editor: Fabio Farinati

Copyright (C) 2017 Sung Eun Kim et al. This is an open access article distributed under the Creative Commons Attribution License, which permits unrestricted use, distribution, and reproduction in any medium, provided the original work is properly cited.

\begin{abstract}
Objectives. Terlipressin is safely used for acute variceal bleeding. However, side effects, such as hyponatremia, although very rare, can occur. We investigated the development of hyponatremia in cirrhotic patients who had acute variceal bleeding treated with terlipressin and the identification of the risk factors associated with the development of hyponatremia. Design and Methods. This retrospective, case-control study investigated 88 cirrhotic patients who developed hyponatremia and 176 controls that did not develop hyponatremia and were matched in terms of age and gender during the same period following terlipressin administration. Results. The overall change in serum sodium concentration and the mean lowest serum sodium concentration were $3.44 \pm 9.55$ and $132.44 \pm 8.78 \mathrm{mEq} / \mathrm{L}$ during treatment, respectively. Multivariate analysis revealed that baseline serum sodium was an independent positive predictor, and the presence of baseline serum creatinine, HBV, DM, creatinine, and shock on admission was independent negative predictors of hyponatremia $(P<0.05)$. Conclusion. The presence of HBV, DM, the baseline serum sodium, shock on admission, and especially baseline creatinine may be predictive of the development of hyponatremia after terlipressin treatment. Therefore, physicians conduct vigilant monitoring associated with severe hyponatremia when cirrhotic patients with preserved renal function are treated with terlipressin for variceal bleeding.
\end{abstract}

\section{Introduction}

The mortality from variceal bleeding in the patients with cirrhosis has decreased from approximately $50 \%$ to $15-20 \%$ due to the development of endoscopic treatments and the early administration of portal pressure-reducing drugs $[1,2]$. Variceal bleeding is a lethal complication of cirrhosis that results from portal hypertension. Terlipressin is a synthetic analogue of vasopressin that is effective in controlling acute variceal bleeding [3-6] and is considered to be a safe vasoconstrictor because terlipressin exhibited increased selectivity for the V1 receptor compared with arginine vasopressin. However, several studies have reported patients with variceal bleeding who developed mild to 
severe hyponatremia upon treatment with standard doses of terlipressin [7-10]. Furthermore, most recent practice guidelines for the management of gastrointestinal bleeding due to portal hypertension recommended that hyponatremia has been occurred in patients under terlipressin therapy, especially in patients with preserved liver function [11]. We also experienced a case of hyponatremic seizure induced by treatment with standard doses of terlipressin in a 52-year-old female who had isolated gastric variceal bleeding secondary to alcoholic liver cirrhosis [12]. Therefore, we thought that the development of hyponatremia may be an important side effect in cirrhotic patients with acute variceal bleeding following treatment with terlipressin. Moreover, there are no reports of the development of hyponatremia after the administration of terlipressin for acute variceal bleeding in patients with hepatocellular carcinomas (HCCs).

Thus, we investigated the development of hyponatremia in cirrhotic patients with and without HCC who had acute variceal bleeding that was treated with the standard doses of terlipressin and attempted to identify the risk factors associated with the development of hyponatremia.

\section{Materials and Methods}

2.1. Patients and Study Design. A total of 88 cirrhotic patients with or without HCC who developed hyponatremia following the administration of terlipressin (Ferring International, Saint-Prex, Switzerland) for acute variceal bleeding were retrospectively enrolled from the Hallym University Sacred Heart Hospital, Korea, from January 2007 to March 2012. For the purpose of comparison, 176 cirrhotic patients with or without HCC who had not developed hyponatremia and who matched the patients who developed hyponatremia in age and gender were selected during the same period. The diagnosis of cirrhosis was based on clinical evidence of portal hypertension (the presence of esophagogastric varices and/or ascites plus splenomegaly with a platelet count $<100,000 / \mathrm{mm}^{3}$ ) or ultrasonography or computed tomography features suggesting cirrhosis, including liver surface irregularities and nodularities [13]. HCC was diagnosed according to the noninvasive diagnostic criteria of the American Association for the Study of Liver Diseases or histopathological examination [14].

Acute variceal bleeding was defined as a diagnosis made by an endoscopist with no other defined cause of bleeding. Endoscopy was performed when a cirrhotic patient with or without HCC exhibited a clinical suspicion of bleeding, such as hematemesis, melena, hematochezia, or an unexplained hemoglobin decrease of $>2 \mathrm{~g} / \mathrm{dL}$. Endoscopic treatments, such as endoscopic variceal band ligation and sclerotherapy for variceal bleeding, were performed at the time of diagnosis in most patients. All patients were initially intravenously injected with $2 \mathrm{mg}$ terlipressin every 4 hours and subsequently injected with additional $1 \mathrm{mg}$ doses every 4 hours for a total of 5 days [3-6]. Hyponatremia was defined as a decrease in sodium concentration $>5 \mathrm{mmol} / \mathrm{L}$. None of the patients had syndrome for inappropriate antidiuretic hormone, pneumonia, pulmonary tuberculosis, small cell lung cancer, or infections of the central nervous system. The study protocol was reviewed and approved by the institutional review board of Hallym University Sacred Heart (HUSHHIRB/EC2016-I014), and the study was conducted in accordance with the ethical guidelines of the 1975 Declaration of Helsinki.

2.2. Clinical and Laboratory Assessment. The baseline serum sodium concentration was taken as that observed on admission, and the lowest level of sodium concentration obtained between 2 and 5 days after terlipressin administration was recorded. All patients were subjected to hepatic and renal function tests, cell blood counts, and daily serum electrolyte tests during the period of terlipressin treatment. Cell blood counts, hepatic and renal function tests, and serum electrolyte tests were performed every 2-3 days until discharge or death following the end of terlipressin administration.

2.3. Statistical Analyses. The statistical analyses were performed using SPSS version 18.0 (SPSS Inc., Chicago, IL, USA). All data are expressed as the means \pm the SD or the medians with the ranges. The continuous data were analyzed with Student's $t$-tests for equal variance or Mann-Whitney tests for unequal variance, and categorical valuables were investigated with Pearson's $\chi^{2}$ tests. Univariate and multivariate logistic regression model analyses were utilized to determine the independent predictors of the development of hyponatremia and the main factors associated with in-hospital mortality. $P<0.05$ was considered to indicate significance.

\section{Results}

3.1. Patient Characteristics. The baseline characteristics of all of the patients $(n=264)$ are summarized in Table 1. Among these patients, 88 patients who developed hyponatremia were included as the case group, and 176 patients who were matched for age and sex were randomly selected as the control group using a computer-generated random number table. In the case group, seventy-three (83\%) patients were male, and the mean age was 56.3 years. In the control group, one hundred forty-six (83\%) patients were male, and the mean age was 57.5 years. All enrolled patients had cirrhosis. The prevalence of hepatitis B surface antigen positivity and diabetes mellitus (DM) was significantly higher in the control group than in the case group ( $47.2 \%$ versus $30.7 \% ; P=0.011$; and $47.9 \%$ versus $19.3 \% ; P=0.029)$. The Child-Pugh score and the Model for End-Stage Liver Disease (MELD) score were used to assess decreased liver function, and both were significantly higher in the control group than in the case group $(9.5 \pm 2.7$ versus $8.4 \pm 2.3 ; P=0.001,20.7 \pm 10.2$ versus $14.0 \pm 5.0 ; P<0.001)$. The serum levels of creatinine were significantly higher in the control group compared with the case group $(1.6 \pm 1.4 \mathrm{mg} / \mathrm{dL}$ versus $0.8 \pm 0.4 \mathrm{mg} / \mathrm{dL} ; P<0.001)$. The baseline sodium levels were $137.8 \pm 4.5 \mathrm{mmol} / \mathrm{L}$ in the case group and $134.1 \pm 7.1 \mathrm{mmol} / \mathrm{L}$ in the control group $(P<0.001)$. The lowest sodium levels after 
TABLE 1: Baseline characteristics of the study population.

\begin{tabular}{|c|c|c|c|}
\hline Characteristics & Hyponatremia $(n=88)$ & Control $(n=176)$ & $P$ value \\
\hline Age (years) & $56.3 \pm 10.3$ & $57.5 \pm 11.3$ & 0.421 \\
\hline Males, $n(\%)$ & $73(83)$ & $146(83)$ & 1.000 \\
\hline \multicolumn{4}{|l|}{ Etiology of cirrhosis, $n(\%)$} \\
\hline Alcohol & $55(62.5)$ & $97(55.1)$ & 0.252 \\
\hline Hepatitis B virus & $27(30.7)$ & $83(47.2)$ & 0.011 \\
\hline Hepatitis C virus & $6(6.8)$ & $6(4.5)$ & 0.437 \\
\hline \multicolumn{4}{|c|}{ Concomitant complications of cirrhosis, $n(\%)$} \\
\hline Ascites & $66(75)$ & $123(69.9)$ & 0.385 \\
\hline Hepatic encephalopathy & $18(20.5)$ & $46(26.1)$ & 0.310 \\
\hline Spontaneous bacterial peritonitis & $3(3.4)$ & $15(8.5)$ & 0.120 \\
\hline Hepatocellular carcinoma, $n(\%)$ & $26(29.5)$ & $51(29)$ & 0.924 \\
\hline BCLC stage A/B/C/D, $n(\%)$ & $7 / 6 / 3 / 10(27 / 23 / 12 / 38)$ & $8 / 7 / 11 / 25(16 / 14 / 22 / 48)$ & 0.336 \\
\hline Diabetes mellitus, $n(\%)$ & $17(19.3)$ & $(47.9)$ & 0.029 \\
\hline Hypertension, $n(\%)$ & $14(15.9)$ & $16(9.1)$ & 0.093 \\
\hline Hemoglobin, g/dL & $9.5 \pm 2.0$ & $9.2 \pm 8.2$ & 0.822 \\
\hline Bilirubin, mg/dL & $2.3 \pm 1.9$ & $5.6 \pm 7.6$ & $<0.001$ \\
\hline Albumin, g/dL & $3.0 \pm 0.6$ & $2.8 \pm 0.6$ & 0.006 \\
\hline INR & $1.6 \pm 0.5$ & $2.0 \pm 1.2$ & 0.001 \\
\hline Creatinine, $\mathrm{mg} / \mathrm{dL}$ & $0.8 \pm 0.4$ & $1.6 \pm 1.4$ & $<0.001$ \\
\hline Initial sodium, $\mathrm{mEq}$ & $137.8 \pm 4.5$ & $134.1 \pm 7.2$ & $<0.001$ \\
\hline Lowest sodium, $\mathrm{mEq}$ & $125.9 \pm 6.8$ & $137.2 \pm 7.8$ & $<0.001$ \\
\hline MELD score & $14.0 \pm 5.0$ & $20.7 \pm 10.2$ & $<0.001$ \\
\hline Child-Pugh score & $8.3 \pm 2.3$ & $9.5 \pm 2.7$ & 0.001 \\
\hline Shock on admission, $n(\%)$ & $4(4.5)$ & $45(34.3)$ & $<0.001$ \\
\hline Blood transfusion (packed red cells) & $3.2 \pm 2.2$ & $3.9 \pm 3.6$ & 0.118 \\
\hline Cumulative dose of terlipressin (mg) & $17.9 \pm 3.8$ & $17.6 \pm 7.5$ & 0.621 \\
\hline Amount of free water hydration (L) & $4.7 \pm 2.3$ & $4.05 \pm 3.9$ & 0.142 \\
\hline
\end{tabular}

BCLC: Barcelona Clinic Liver Cancer; INR: international normalized ratio; MELD: Model for End-Stage Liver Disease; the data are expressed as the means \pm the standard deviations (ranges) or numbers (\%).

terlipressin treatment were $125.9 \pm 6.8 \mathrm{mmol} / \mathrm{L}$ in the case group and $137.2 \pm 7.8 \mathrm{mmol} / \mathrm{L}$ in the control group $(P<0.001)$. There was statistically insignificant difference between the groups in terms of age, sex, alcohol consumption, hepatitis $\mathrm{C}$ virus (HCV) antibody positivity, concomitant complications of cirrhosis, HCC including stages of Barcelona Clinic Liver Cancer (BCLC), hypertension, hemoglobin level, the amount of blood transfusion, cumulative dose of terlipressin, and the amount of free water hydration.

3.2. Changes in Serum Sodium Concentration. The overall change in serum sodium concentration and the mean lowest serum sodium concentration were $3.4 \pm 9.6 \mathrm{mmol} / \mathrm{L}$ and $132.4 \pm 8.8 \mathrm{mmol} / \mathrm{L}$ during treatment, respectively.

To determine the factors associated with the development of hyponatremia during terlipressin treatment, eightyeight patients with hyponatremia were categorized into the three groups described above (Table 2).

Forty-seven patients (53.5\%) exhibited mild decreases (between 5 and $10 \mathrm{mmol} / \mathrm{L}$ ), 20 patients (22.7\%) exhibited moderate decreases (between 10 and $15 \mathrm{mmol} / \mathrm{L}$ ), and 21 patients (23.8\%) exhibited severe decreases in serum sodium (greater than $15 \mathrm{mmol} / \mathrm{L}$ ). The mean baseline serum sodium concentrations in the groups of mild, moderate, and severe decreases were $137.28,136.96$, and $139.9 \mathrm{mmol} / \mathrm{L}$, respectively. The mean lowest serum sodium concentrations in the groups of mild, moderate, and severe decreases were $129.98,124.25$, and $118.57 \mathrm{mmol} / \mathrm{L}$, respectively. The majority of patients who developed hyponatremia exhibited recoveries toward baseline serum sodium concentration after the end of treatment. The recovery time of the serum sodium concentration was $2.5 \pm 1.8$ days after the end of treatment. The patients who developed hyponatremia had higher baseline serum sodium levels than the patients in the other groups. There were inverse associations between the changes in the baseline serum bilirubin, MELD score, and Child-Pugh score and the changes in the serum sodium levels. The patient who developed severe hyponatremia exhibited the lower serum bilirubin levels, lower MELD scores, and lower Child-Pugh scores relative to the patients who developed mild hyponatremia, who had the highest serum bilirubin levels, the highest MELD scores, and the highest ChildPugh scores. There were no significant differences between 
TABLE 2: Characteristics of the patients categorized according to changes in serum sodium concentration.

\begin{tabular}{|c|c|c|c|c|}
\hline & $\Delta$ sodium $5-10 \mathrm{mEq}(n=47)$ & $\Delta$ sodium $10-15 \mathrm{mEq}(n=20)$ & $\Delta$ sodium $>15 \mathrm{mEq}(n=21)$ & $P$ value \\
\hline Ascites & $32(68)$ & $19(95)$ & $15(71)$ & 0.460 \\
\hline Hepatic encephalopathy & $7(15)$ & $7(35)$ & $4(19)$ & 0.462 \\
\hline Hemoglobin, g/dL & $9.5 \pm 1.9$ & $9.7 \pm 2.1$ & $9.2 \pm 2.3$ & 0.766 \\
\hline Bilirubin, mg/dL & $2.4 \pm 2.1$ & $2.9 \pm 1.9$ & $1.4 \pm 0.7$ & 0.024 \\
\hline Albumin, g/dL & $3.0 \pm 0.6$ & $3.0 \pm 0.5$ & $3.1 \pm 0.6$ & 0.575 \\
\hline INR & $1.6 \pm 0.5$ & $1.7 \pm 0.6$ & $1.5 \pm 0.2$ & 0.275 \\
\hline Creatinine, $\mathrm{mg} / \mathrm{dL}$ & $0.8 \pm 0.4$ & $0.9 \pm 0.4$ & $0.8 \pm 0.3$ & 0.632 \\
\hline Initial sodium, mEq/ & $137.3 \pm 4.0$ & $137.0 \pm 4.5$ & $140.0 \pm 5.0$ & 0.05 \\
\hline MELD score & $14.0 \pm 5.3$ & $16.0 \pm 5.9$ & $12.2 \pm 2.4$ & 0.059 \\
\hline Child-Pugh score & $8.3 \pm 2.5$ & $9.4 \pm 2.1$ & $7.5 \pm 1.1$ & 0.031 \\
\hline Blood transfusion (packed red cells) & $3.3 \pm 2.3$ & $3.2 \pm 2.3$ & $3.1 \pm 1.9$ & 0.963 \\
\hline Cumulative terlipressin dose (mg) & $18.0 \pm 4.4$ & $17.6 \pm 3.6$ & $18.0 \pm 2.6$ & 0.932 \\
\hline Amount of free water hydration (L) & $4.3 \pm 2.3$ & $5.4 \pm 2.6$ & $5.0 \pm 1.9$ & 0.134 \\
\hline
\end{tabular}

INR: international normalized ratio; MELD: Model for End-Stage Liver Disease; the data are expressed as the means \pm the standard deviations (ranges) or numbers $(\%)$

groups with respect to the previous histories of ascites and hepatic encephalopathy, baseline hemoglobin, baseline serum albumin, international normalized ratio, serum creatinine, blood transfusion amount, cumulative terlipressin dose, or the amount of free water hydration.

One patient developed mental changes with seizure coincidence with a marked reduction in serum sodium levels from 135 to $114 \mathrm{mmol} / \mathrm{L}$ within the first 48 hours of terlipressin treatment. This patient did not exhibit any abnormalities on brain computed tomography or electroencephalogram. The serum sodium levels and mental status of this patient did not improve despite the administration of 3\% hypertonic saline. However, this patient successfully recovered the serum sodium concentration and mental status within 36 hours after terlipressin withdrawal.

\subsection{Predictive Factors for the Development of Hyponatremia} following Terlipressin Treatment. Univariate and multivariate logistic regression models were used to identify the independent risk factors that were significantly associated with development of hyponatremia during terlipressin treatment. In the univariate analysis, hepatitis $\mathrm{B}$ virus ( $\mathrm{HBV}$ ) positivity, DM, baseline serum creatinine level, baseline serum sodium level, baseline Child-Pugh score, and shock on admission were the candidate variables that were identified for the multivariate analysis $(P<0.05)$. Other clinical factors, such as age, gender, alcohol consumption, infectivity of $\mathrm{HCV}$, ascites, hepatic encephalopathy, spontaneous bacterial peritonitis, HCC, hypertension, cumulative dose of terlipressin, baseline hemoglobin level, and the amount of free water hydration, were not significantly associated with the development of hyponatremia during terlipressin treatment. In the multivariate analysis, baseline normal or near-normal serum sodium and creatinine levels were independent positive predictors of the development of hyponatremia. The presence of HBV, $\mathrm{DM}$, and shock on admission was independent negative predictors of the development of hyponatremia $(P<0.05)$. The strongest predictor of the development of hyponatremia following terlipressin treatment was the baseline serum creatinine level (odds ratio [OR], 0.22; 95\% confidence interval [CI], 0.10-0.46; $P<0.001$; Table 3).

3.4. In-Hospital Mortality. Overall, 69 of the 264 patients (26.1\%) died during hospitalization. The in-hospital mortality rate was significantly lower in the group that developed hyponatremia than in the control group $(6.8 \%$ versus $35.8 \% ; P<0.001)$. In the univariate analysis, HBV infection, alcohol, hepatic encephalopathy, HCC, baseline serum sodium level, serum bilirubin, serum albumin, international normalized ratio (INR), baseline serum creatinine level, baseline Child-Pugh score, baseline MELD score, shock on admission, the amount of blood transfusion, and the development of hyponatremia after terlipressin treatment were the candidate variables that were identified for the multivariate analysis $(P<0.05)$. Other clinical factors, such as age, gender, HCV infection, ascites, spontaneous bacterial peritonitis, hypertension, DM, hemoglobin level, and the cumulative dose of terlipressin, were not found to be significantly associated with in-hospital mortality. In the multivariate analysis, the development of hyponatremia after terlipressin treatment (OR, 0.19; 95\% CI, 0.07-0.61; $P=0.005)$, HCC (OR, 8.39; 95\% CI, 2.81-25.05; $P<0.001$ ), and baseline serum bilirubin (OR, 1.08; 95\% CI, 1.01-1.15; $P<0.001$ ) was found to be independent predictors of in-hospital mortality (Table 4).

\section{Discussion}

Recent studies have demonstrated that terlipressin treatment for variceal bleeding results in the development of hyponatremia in $35-67 \%$ of cirrhotic patients $[9,10]$. However, other randomized studies have reported that patients with acute variceal bleeding develop hyponatremia at rates of $0 \%$ and 6\% [15-18]. This difference might have been influenced by differences in the definitions and methods for the identification of hyponatremia and selection bias. Because the 
TABLE 3: Univariate and multivariate logistic analyses of the risk factors for the development of hyponatremia.

\begin{tabular}{|c|c|c|c|c|c|c|}
\hline & \multicolumn{3}{|c|}{ Univariate analysis } & \multicolumn{3}{|c|}{ Multivariate analysis } \\
\hline & OR & $95 \% \mathrm{CI}$ & $P$ value & OR & $95 \%$ CI & $P$ value \\
\hline Hepatitis B virus positivity & 0.50 & $0.29-0.85$ & 0.011 & 0.54 & $0.29-0.99$ & 0.05 \\
\hline Diabetes mellitus & 0.50 & $0.27-0.93$ & 0.027 & 0.47 & $0.23-0.93$ & 0.03 \\
\hline Creatinine & 0.17 & $0.08-0.35$ & $<0.001$ & 0.22 & $0.10-0.46$ & $<0.001$ \\
\hline Initial sodium & 1.11 & $1.06-1.16$ & $<0.001$ & 1.09 & $1.03-1.17$ & 0.005 \\
\hline Child-Pugh score & 0.83 & $0.75-0.93$ & 0.001 & 1.06 & $0.91-1.23$ & 0.441 \\
\hline Shock on admission & 0.14 & $0.05-0.40$ & $<0.001$ & 0.19 & $0.06-0.61$ & 0.005 \\
\hline
\end{tabular}

TABLE 4: Univariate and multivariate logistic analyses of the risk factors for the in-hospital mortality.

\begin{tabular}{|c|c|c|c|c|c|c|}
\hline & \multicolumn{3}{|c|}{ Univariate analysis } & \multicolumn{3}{|c|}{ Multivariate analysis } \\
\hline & OR & $95 \%$ CI & $P$ value & OR & $95 \%$ CI & $P$ value \\
\hline Hepatitis B virus positivity & 2.27 & $1.30-3.97$ & 0.031 & 0.38 & $0.14-1.08$ & 0.069 \\
\hline Alcohol & 0.50 & $0.29-0.87$ & 0.014 & 0.45 & $0.19-1.08$ & 0.075 \\
\hline Hepatocellular carcinoma & 3.43 & $1.92-6.14$ & $<0.001$ & 8.39 & $2.81-25.05$ & $<0.001$ \\
\hline Hepatic encephalopathy & 3.64 & $1.99-6.66$ & $<0.001$ & 1.61 & $0.69-3.78$ & 0.273 \\
\hline Bilirubin & 1.14 & $1.09-1.20$ & $<0.001$ & 1.08 & $1.01-1.15$ & 0.017 \\
\hline Albumin & 0.32 & $0.18-0.54$ & $<0.001$ & 0.95 & $0.42-2.17$ & 0.907 \\
\hline INR & 3.02 & $2.00-4.55$ & $<0.001$ & 1.64 & $0.94-2.89$ & 0.08 \\
\hline Creatinine & 1.83 & $1.37-2.44$ & $<0.001$ & 1.18 & $0.90-1.55$ & 0.229 \\
\hline Initial sodium & 0.90 & $0.86-0.94$ & $<0.001$ & 0.97 & $0.91-1.02$ & 0.248 \\
\hline MELD score & 1.15 & $1.11-1.20$ & $<0.001$ & & & \\
\hline Child-Pugh score & 1.55 & $1.36-1.76$ & $<0.001$ & & & \\
\hline Development of hyponatremia & 0.13 & $0.05-0.32$ & $<0.001$ & 0.19 & $0.07-0.61$ & 0.005 \\
\hline Shock on admission & 5.66 & $2.92-10.95$ & $<0.001$ & 1.06 & $0.94-1.20$ & 0.369 \\
\hline Blood transfusion (PRCs) & 1.10 & $1.01-1.19$ & 0.028 & 1.06 & $0.94-1.19$ & 0.369 \\
\hline
\end{tabular}

INR: international normalized ratio; MELD: Model for End-Stage Liver Disease. PRCs: packed red cells.

present study was a matched case-control study, we were unable to precisely compare the rates of the occurrence of hyponatremia. However, we were able to evaluate the risk factors for the development of hyponatremia in cirrhotic patients with terlipressin treatment. The multivariate analysis of the development of hyponatremia indicated that the presence of HBV, DM, baseline serum creatinine levels, and shock on admission was independent negative predictors of the development of hyponatremia and that baseline normal or near-normal serum sodium was independent positive predictor of the development of hyponatremia. Previous studies have demonstrated that lower MELD scores [9] and higher baseline serum sodium levels $[9,10]$ are important predictors of hyponatremia. The present study revealed similar results in that higher baseline serum sodium levels and relatively preserved liver function were associated with the development of hyponatremia. Interestingly, our results strongly suggested that the baseline serum creatinine level is the most important predictor of reductions in sodium level (OR, 0.22; 95\% CI, $0.10-0.46 ; P<0.001)$. Terlipressin is an effective drug for variceal bleeding and hepatorenal syndrome in cirrhosis because it vasoconstricts the splanchnic circulation via the V1 receptors [19], but vasopressin also exerts activity on the renal V2 receptors present in the renal collecting ducts; therefore, it causes antidiuresis and natriuresis $[20,21]$. It is well known that in patients with hepatorenal syndrome, terlipressin is able to increase serum sodium concentration toward an improvement in renal perfusion and water excretion. However, the administration of single dose of terlipressin to patients with cirrhosis is associated with an increase in aquaporin-2 excretion and a reduction in solute-free water clearance, and these findings are consistent with the significant activation of the renal V2 receptors and antidiuretic actions in patients with cirrhosis [22]. Therefore, the underlying renal function may be an important factor in the reduction in serum sodium because the renal V2 receptors are preoccupied by endogenous vasopressin when patients have poor underlying renal function [10]. The present study revealed that HBV positivity was negatively associated with the development of hyponatremia after terlipressin treatment (OR, 0.54; 95\% CI, 0.29-0.99; $P=0.05$ ). HBV infection can cause $\mathrm{HBV}$-associated glomerulonephritis, which is the most common extrahepatic disorder of chronic $\mathrm{HBV}$ infection due to immune complex-mediated injuries $[23,24]$. Recently, HBV infection was found to promote apoptotic damage in human renal tubular cells by triggering a pathway of Fas upregulation [25] and to potentially affect renal V2 receptors in renal tubular cells [25]. The present 
study also demonstrated that DM was negatively associated with the development of hyponatremia after terlipressin treatment (OR, 0.47; 95\% CI, 0.23-0.93; $P=0.03$ ). Diabetic renal injury, which is one of the most important chronic renal diseases in the world, may also exhibit protective effects against the development of hyponatremia for the same reason this effect is present in HBV infection. Shock on admission was negatively associated with the development of hyponatremia (OR, 0.19; 95\% CI, 0.06-0.61; $P=0.005$ ). Shock has been identified as a common cause of acute kidney injury [26]. Ultimately, the development of hyponatremia in cirrhotic patients with variceal bleeding following terlipressin administration was mainly associated with renal function and other factors that influence renal function, although previous studies have not revealed associations between renal function and the development of hyponatremia due to small numbers of patients and retrospective designs $[9,10]$.

In the present study, the development of hyponatremia following terlipressin treatment (OR, 0.19; 95\% CI, 0.07-0.61; $P=0.005$ ), HCC (OR, 8.39; 95\% CI, 2.81-25.05; $P<0.001$ ), and baseline serum bilirubin level (OR, 1.08; 95\% CI, 1.01-1.15; $P=0.017)$ was associated with inhospital mortality. As expected, liver function at admission was a risk factor for in-hospital mortality. Indeed, because other studies have not enrolled patients with HCC, these studies were unable to describe the role of HCC in mortality or the occurrence of hyponatremia. However, in clinical settings, because HCC with and without portal invasion is the main cause of variceal bleeding, the present study was able to provide data regarding terlipressin treatment in HCC patients with variceal bleeding. The patients who developed hyponatremia after terlipressin treatment tended to exhibit lower in-hospital mortality, which may have been associated with the relatively preserved liver and renal functions of these patients who have been described above.

One of the 21 patients with severe hyponatremia developed seizures. This patient exhibited rapid recovery of the serum sodium level and the neurologic abnormalities without sequelae after the withdrawal of terlipressin administration. However, the serum sodium levels and mental status of the patient did not improve before the withdrawal of terlipressin administration despite the administration of $3 \%$ hypertonic saline. There have been several reports of cases of hyponatremia with seizure that have rapidly recovered nearly normal sodium levels within a day of the withdrawal of terlipressin $[7,8,12]$. However, Solà et al. reported on a patient with severe hyponatremia and seizure who developed an osmotic demyelination syndrome after a very rapid recovery of the serum sodium level secondary to the withdrawal of terlipressin and treatment with hypertonic saline [9]. Therefore, if cirrhotic patients with relatively healthy hepatic and renal conditions exhibit variceal bleeding following terlipressin therapy with mental changes, physicians should be suspicious of the development of hyponatremia, although the development of hyponatremia following terlipressin treatment is rapidly reversible and is not associated with in-hospital mortality.

The present study has several limitations. First, enrolled patients were heterogeneous because we did not exclude patients with HCC. However, the presence or absence of HCC was not associated with the development of hyponatremia, and this finding might suggest that HCC was not related to the development of hyponatremia after terlipressin administration. Second, this was a retrospective matched case-control design study. Thus, future large-scale prospective studies are needed. Despite these limitations, to our knowledge, the present study is the first to report an association between baseline renal function and hyponatremia following terlipressin therapy in the patients with cirrhosis and other factors that influence renal function.

In conclusion, terlipressin treatment is commonly applied in cases of variceal bleeding but may often cause hyponatremia. The presence of $\mathrm{HBV}, \mathrm{DM}$, the baseline serum sodium level, shock on admission, and particularly baseline renal function may be predictive of the development of hyponatremia after terlipressin treatment. The role of renal dysfunction and baseline hyponatremia as potential protective factors against terlipressin-induced hyponatremia should be further developed. Although the development of hyponatremia after terlipressin treatment is rapidly reversible and is not associated with in-hospital mortality, physicians closely monitor electrolytes daily to prevent the possible neurological complications associated with severe hyponatremia when cirrhotic patients with relatively preserved liver function and renal conditions who exhibit variceal bleeding are treated with terlipressin.

\section{Abbreviations \\ HCC: Hepatocellular carcinoma \\ DM: Diabetes mellitus \\ MELD: Model for End-Stage Liver Disease \\ HCV: Hepatitis $\mathrm{C}$ virus \\ HBV: Hepatitis B virus \\ OR: $\quad$ Odds ratio \\ CI: Confidence interval \\ INR: International normalized ratio \\ PRC: Packed red cell.}

\section{Ethical Approval}

The study protocol was reviewed and approved by the institutional review board of Hallym University Sacred Heart (2016-I014), and the study was conducted in accordance with the ethical guidelines of the 1975 Declaration of Helsinki.

\section{Disclosure}

This study was presented before as an abstract at the Liver Week 2016 and Asia Pacific Digestive Week 2016.

\section{Conflicts of Interest}

The authors have declared that no competing interests exist.

\section{Authors' Contributions}

Sung Eun Kim, ChoongKee Park, and Jun Goo Kang did the conception and design. Dong Min Jung, Ji Won Park, 
Bohyun Lee, Jae Seung Soh, Hyun Lim, ChulSik Kim, and SeongJin Lee did the acquisition of data. Sung Eun Kim, Ho Suk Kang, Sung Hoon Moon, Jong Hyeok Kim, and SungHee Ihm did the analysis and interpretation of data. Sung Eun Kim, and Jun Goo Kang did the drafting of the manuscript. All authors read and approved the final manuscript.

\section{Acknowledgments}

This research was supported by the Hallym University Research Fund (HURF-2014-01).

\section{References}

[1] G. D'Amico, R. De Franchis, and Cooperative Study Group, "Upper digestive bleeding in cirrhosis. Post-therapeutic outcome and prognostic indicators," Hepatology, vol. 38, pp. 599-612, 2003.

[2] G. D'Amico, J. C. Garcia-Pagan, A. Luca, and J. Bosch, "Hepatic vein pressure gradient reduction and prevention of variceal bleeding in cirrhosis: a systematic review," Gastroenterology, vol. 131, pp. 1611-1624, 2006.

[3] G. D’Amico, L. Pagliaro, and J. Bosch, "Pharmacological treatment of portal hypertension: an evidence-based approach," Seminars in Liver Disease, vol. 19, pp. 475-505, 1999.

[4] G. Garcia-Tsao and J. Bosch, "Management of varices and variceal hemorrhage in cirrhosis," The New England Journal of Medicine, vol. 362, pp. 823-832, 2010.

[5] R. De Franchis and Baveno V Faculty, "Revising consensus in portal hypertension: report of the Baveno $\mathrm{V}$ consensus workshop on methodology of diagnosis and therapy in portal hypertension," Journal of Hepatology, vol. 53, pp. 762-768, 2010.

[6] G. Garcia Tsao, A. J. Sanyal, N. D. Grace, W. Carey, the Practice Guideline Committee of the American Association for the Study of Liver Diseases, and the Practice Parameters Committee of the American College of Gastroenterology, "Prevention and management of gastroesophageal varices and variceal hemorrhage in cirrhosis," Hepatology, vol. 46, pp. 922-938, 2007.

[7] E. Dunwoodie and S. Jowett, "Terlipressin causing a hyponatraemic seizure," Scandinavian Journal of Gastroenterology, vol. 42, p. 665, 2007.

[8] J. J. Hyun, Y. S. Seo, K. G. Lee et al., “Terlipressin-induced hyponatremic seizure," Scandinavian Journal of Gastroenterology, vol. 45, pp. 501-504, 2010.

[9] E. Solà, S. Lens, M. Guevara et al., "Hyponatremia in patients treated with terlipressin for severe gastrointestinal bleeding due to portal hypertension," Hepatology, vol. 52, pp. 17831790, 2010.

[10] Y. J. Kang, E. J. Bae, K. Hwang et al., "Initial serum sodium concentration determines the decrease in sodium level after terlipressin administration in patients with liver cirrhosis," Springerplus, vol. 2, p. 519, 2013.

[11] R. De Franchis and Baveno VI Faculty, "Expanding consensus in portal hypertension: report of the Baveno VI consensus workshop: stratifying risk and individualizing care for portal hypertension," Journal of Hepatology, vol. 63, pp. 743-752, 2015.

[12] J. H. Kim, J. K. Kim, S. Y. Mun et al., "A case of hyponatraemic seizure following terlipressin therapy for a variceal hemorrhage in a patient with liver cirrhosis," Korean Journal of Medicine, vol. 87, pp. 323-327, 2014.

[13] A. Di Lelio, C. Cestari, A. Lomazzi, and L. Beretta, "Cirrhosis: diagnosis with sonographic study of the liver surface," Radiology, vol. 172, pp. 389-392, 1989.

[14] J. Bruix, M. Sherman, and Practice Guidelines Committee, American Association for the Study of Liver Diseases, "Management of hepatocellular carcinoma," Hepatology, vol. 42, pp. 1208-1236, 2005.

[15] A. Escorsell, L. Ruiz del Arbol, R. Planas et al., "Multicenter randomized controlled trial of terlipressin versus sclerotherapy in the treatment of acute variceal bleeding: the TEST study," Hepatology, vol. 32, pp. 471-476, 2000.

[16] F. Feu, L. Ruiz del Arbol, R. Bañares, R. Planas, and J. Bosch, "Double-blind randomized controlled trial comparing terlipressin and somatostatin for acute variceal hemorrhage. Variceal bleeding study group," Gastroenterology, vol. 111, pp. 1291-1299, 1996.

[17] G. H. Lo, W. C. Chen, H. M. Wang et al., "Low-dose terlipressin plus banding ligation versus low-dose terlipressin alone in the prevention of very early rebleeding of oesophageal varices," Gut, vol. 58, pp. 1275-1280, 2009.

[18] S. Abid, W. Jafri, S. Hamid et al., "Terlipressin vs. octreotide in bleeding esophageal varices as an adjuvant therapy with endoscopic band ligation: a randomized double-blind placebocontrolled trial," The American Journal of Gastroenterology, vol. 104, pp. 617-623, 2009.

[19] C. L. Holmes, D. W. Landry, and J. T. Granton, "Science review: vasopressin and the cardiovascular system part 1receptor physiology," Critical Care, vol. 7, pp. 427-434, 2003.

[20] M. L. Forsling, L. A. Aziz, M. Miller, R. Davies, and B. Donovan, "Conversion of triglycylvasopressin to lysinevasopressin in man," The Journal of Endocrinology, vol. 85, pp. 237-244, 1980.

[21] H. Nádvorníková and O. Schück, "The influence of a single dose of vasopressin analogs on human renal potassium excretion," International Journal of Clinical Pharmacology, Therapy, and Toxicology, vol. 20, pp. 155-158, 1982.

[22] A. Krag, F. Bendtsen, E. Pedersen, N. Holstein-Rathlou, and S. Møller, "Effects of terlipressin on the aquaretic system: evidence of antidiuretic effects," American Journal of Physiology - Renal Physiology, vol. 295, pp. F1295-F1300, 2008.

[23] Chapter 9: infection-related glomerulonephritis," Kidney International Supplements, vol. 2, pp. 200-208, 2012.

[24] R. Bhimma and H. M. Coovadia, "Hepatitis B virus-associated nephropathy," American Journal of Nephrology, vol. 24, pp. 198-211, 2004.

[25] C. L. Deng, X. W. Song, H. J. Liang, C. Feng, Y. J. Sheng, and M. Y. Wang, "Chronic hepatitis B serum promotes apoptotic damage in human renal tubular cells," World Journal of Gastroenterology, vol. 12, pp. 1752-1756, 2006.

[26] H. M. Choi, S. C. Kim, M. G. Kim, S. K. Jo, W. Y. Cho, and H. K. Kim, "Etiology and outcomes of anuria in acute kidney injury: a single center study," Kidney Research and Clinical Practice, vol. 34, pp. 13-19, 2015. 


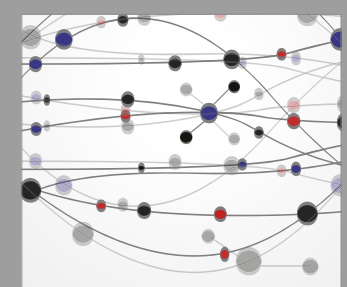

The Scientific World Journal
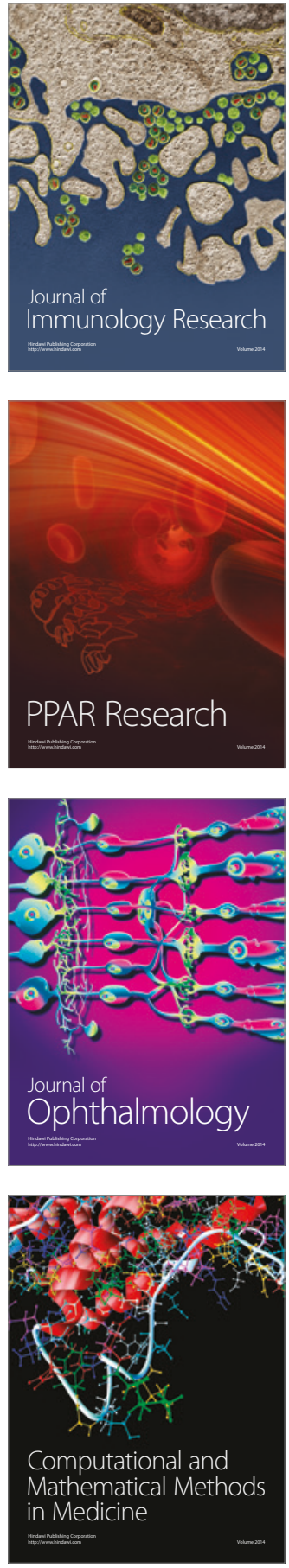

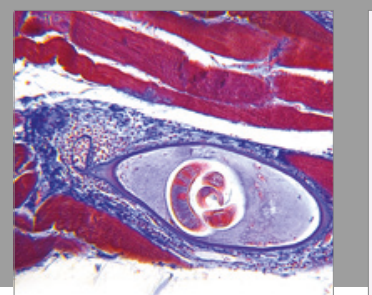

Gastroenterology Research and Practice
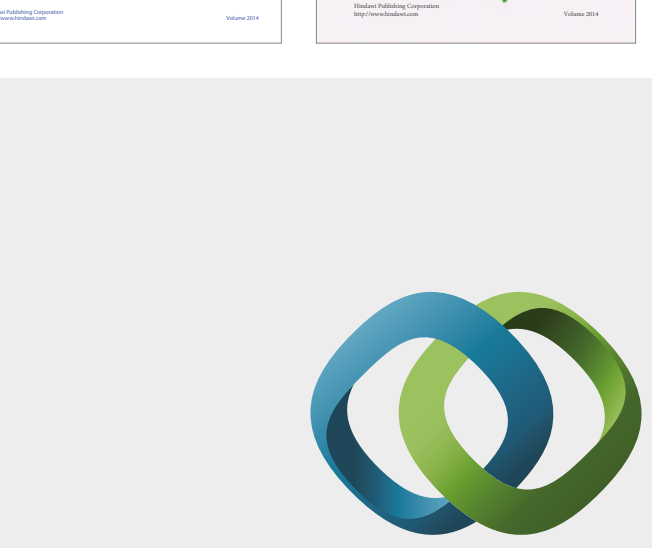

\section{Hindawi}

Submit your manuscripts at

https://www.hindawi.com
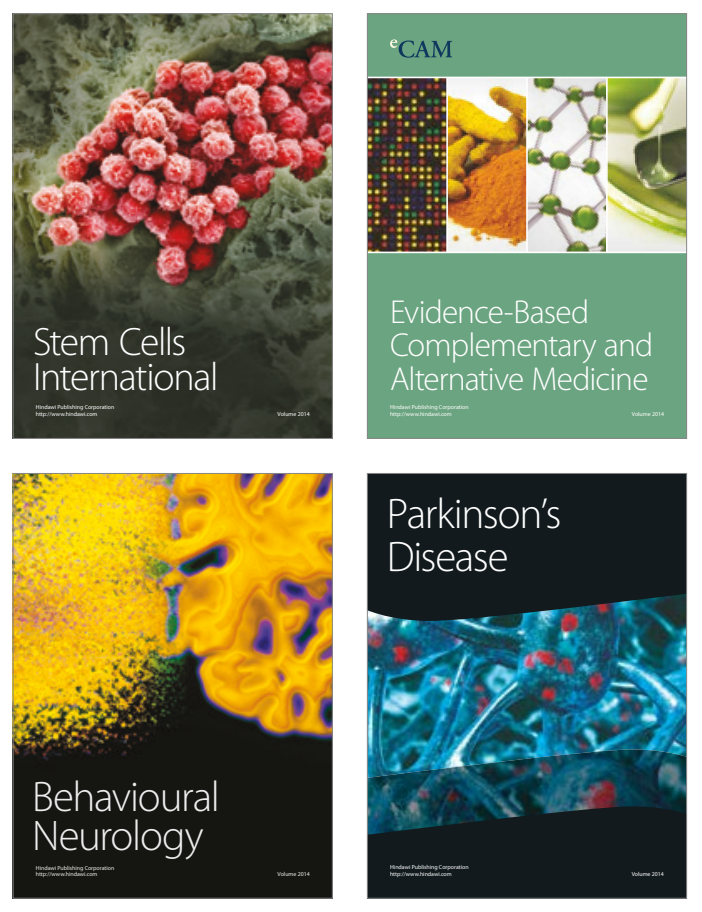
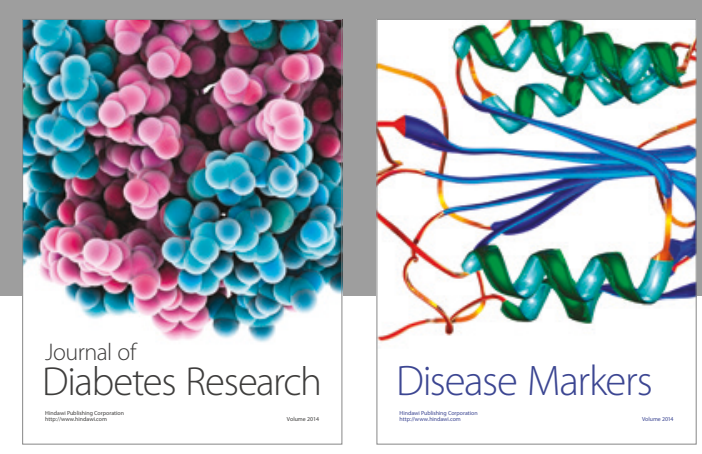

Disease Markers
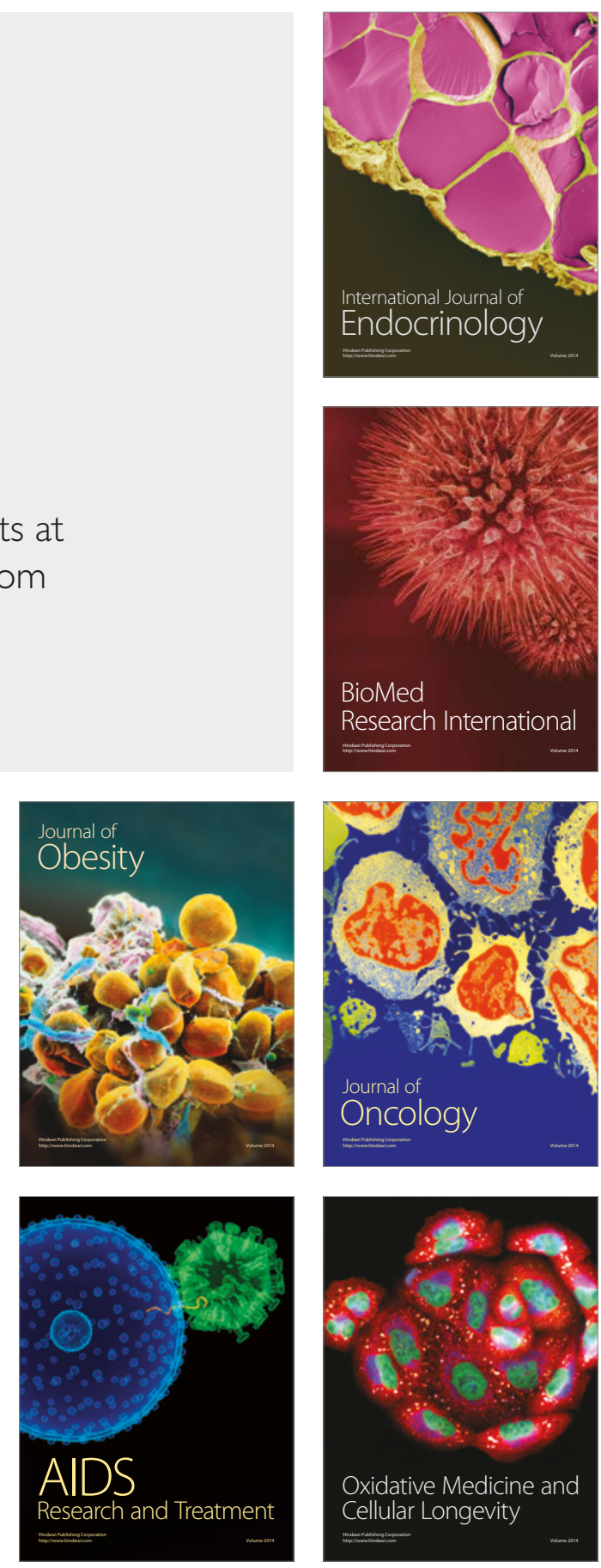